\title{
Femtosecond pump-probe study of the spreading and recurrence of a vibrational wave packet in $\mathrm{Na}_{2}$
}

\author{
T. Baumert, V. Engel, C. Röttgermann, W.T. Strunz and G. Gerber \\ Fakultät für Physik, Albert-Ludwigs-Universität, Hermann-Herder-Strasse 3, W-7800 Freiburg, Germany
}

Received 30 December 1991; in final form 31 January 1992

\begin{abstract}
The motion of a vibrational wave packet in the bound $\mathrm{A}\left({ }^{1} \Sigma_{\mathrm{u}}^{+}\right)$electronic state of the sodium dimer is detected in a femtosecond pump/probe molecular beam experiment. For short times harmonic motion is seen in the total ion yield of $\mathrm{Na}_{2}^{+}$as a function of delay time between the two laser pulses. The spreading of the wave packet results in the loss of the periodic variation of the ion signal. For longer delay times ( $47 \mathrm{ps}$ ) the wave packet regains its initial form which is reflected in the revival structure of the $\mathrm{Na}_{2}^{+}$signal. Time-dependent quantum calculations reproduce the measured effects.
\end{abstract}

\section{Introduction}

Femtosecond techniques have been advanced in recent years to directly probe molecular motions in real time $[1,2]$. It has been shown that the detection of the purely bound state dynamics of a vibrational wave packet can be used to determine the potential energy surface $[3,4]$. The pump pulse passing through the sample prepares a coherent superposition of vibrational states if the spectral width of the pulse is broader than the vibrational spacing of the molecule. As long as spreading is on a longer time scale, the wave packet will propagate back and forth in the bound state potential and this may be reflected in a periodically changing signal as a function of delay time between the pump and the probe pulse. For longer times, spreading becomes effective if the potential is not harmonic. The signal then looses its purely harmonic modulation. However, as time goes on the wave packet will recur and perform a (quasi) harmonic motion again. This effect has been observed in molecular systems with the nuclei undergoing reactive or non-reactive motions [1]. In atoms [5], termed revival, it was found in studies of the dynamics of radial Rydberg wave packets [6-8].

Molecular vibrational wave packet motion has been seen for a number of systems $\left(I_{2}\right.$ [9], NaI $[10,11], I C l[4])$. The signal in the case of $I_{2}$ shows the full recurrence $[3,9]$. The recurrence of an os- cillatory signal is also seen in the predissociation of $\mathrm{NaI}$ [12]. In this case, it has been shown [12-14], that the origin of the NaI recurrence structure is due to the excitation of several resonances with long lifetimes which exist in two strongly coupled potential energy curves (avoided crossing). Therefore the observed recurrence time is much shorter than the expected revival time.

In this Letter, we present data for the $\mathrm{Na}_{2}$ molecule where the bound state motion of a wave packet in the $\mathrm{A}\left({ }^{1} \Sigma_{\mathrm{u}}^{+}\right)$excited state is detected via femtosecond pump/probe ionization spectroscopy $[2,15]$. The excitation scheme of the experiment is indicated in fig. $1 . \mathrm{Na}_{2}$ is excited by the femtosecond pump pulse and a linear combination of eigenstates within the A-state vibrational manifold is prepared. This vibrational wave packet moves in the bound state potential energy surface until the probe pulse interacts with the molecule. The absorption of two probe photons yields $\mathrm{Na}_{2}^{+}$ions in various vibrational states. $\mathrm{A}$ careful analysis $[15,16]$ shows that ions are preferentially formed if the probe pulse is fired at delay times when the wave packet is close to the inner turning point. Resonant two-photon probe transitions at times when the wavefunction is located in the outer turning point region are very unlikely. On the other hand, non-resonant transitions have a much smaller amplitude [16].

There is another ionization pathway since the 


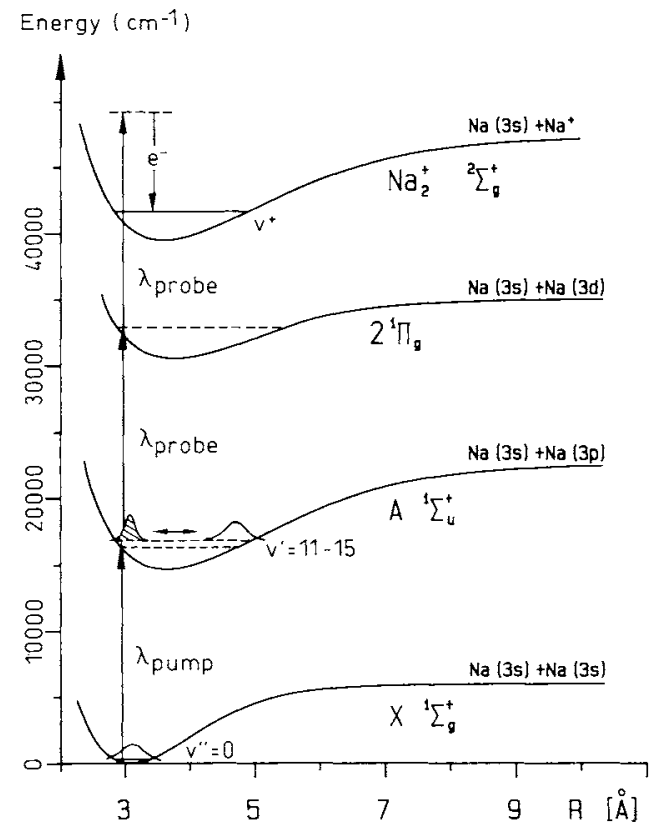

Fig. 1. Excitation scheme for the three-photon pump/probe ionization of $\mathrm{Na}_{2}$ via the $\mathrm{A}^{\mathrm{I}} \Sigma_{\mathrm{u}}^{+}$state.

pump pulse prepares a vibrational wave packet in the $2{ }^{1} \Pi_{\mathrm{g}}$ state as well. This originates from a twophoton transition from the electronic ground state. In this case ions are formed by a one-photon probe transition. As discussed in detail elsewhere [15,16] this process has (for moderate field strength) a rather small probability and gives only a time independent signal. However, for higher laser intensities a second ionization process including a doubly excited Rydberg state contributes strongly to the observed transient $\mathrm{Na}_{2}^{+}$signal [15]. In the present paper we will concentrate on the A-state wave packet motion which is dominant in the observed ionization signal.

\section{Experiment}

The experimental setup is described in detail in ref. [17]. Here we summarize the main features of our technique. A supersonic beam of sodium dimers is produced by expanding pure sodium from an oven operated at $1000 \mathrm{~K}$ through a small nozzle of $100 \mu \mathrm{m}$ diameter. This prepares $\mathrm{Na}_{2}$ in its electronic $\mathrm{X}\left({ }^{1} \Sigma_{\mathrm{g}}^{+}\right)$ground state and predominantly in the vibrational ground state $v^{\prime \prime}=0$.

Molecular ions are formed by a pump and time delayed probe femtosecond laser pulse interacting with the $\mathrm{Na}_{2}$ molecular beam. The femtosecond pulses are generated in a home built colliding pulse mode-locked (CPM) ring dye laser following the design of Valdmanis and Fork [18]. Since the CPM pulse energies are only $\approx 100 \mathrm{pJ}$ we employed a bowtie amplifier pumped by a $308 \mathrm{~nm}$ excimer laser synchronized to the CPM. The recompressed laser pulses are characterized by a central wavelength of $618 \mathrm{~nm}$ and a temporal width of $65 \mathrm{fs} \mathrm{sech}^{2}$. The intensity of the pump and probe laser pulses was adjusted in a way that the average ion signal produced by each of the pulses is reduced to less than $10 \%$. For the pump/ probe measurements the pulses are delayed with respect to each other with a Michelson arrangement which allows for continuous variation of the delay time. The pump and probe pulses enter the interaction region collinearly with the same polarization and perpendicular to the molecular beam. Molecular ions are detected with a TOF spectrometer arranged perpendicular to the beam. Each experimental point was obtained by continuous averaging over 300 laser shots.

\section{Theory}

The theoretical treatment for the present multiphoton ionization experiment will be given in ref. [16]. Here, we briefly summarize the general approach. Time dependent perturbation theory is used to calculate the total ion signal as a function of delay time between pump and probe pulses. The wavefunction created by the pump pulse can be written, to first order, $(\hbar=1)$ as

$$
\begin{aligned}
& |\psi(t), \mathrm{A}\rangle \\
& \quad=\int_{0}^{t} \mathrm{~d} t^{\prime} U_{\mathrm{A}}\left(t-t^{\prime}\right) V_{\mathrm{AX}}\left(t^{\prime}, \omega\right) U_{\mathrm{X}}\left(t^{\prime}\right)\left|\psi_{0}, \mathrm{X}\right\rangle .
\end{aligned}
$$

Here $\left|\psi_{0}, X\right\rangle$ denotes the vibrational ground state wavefunction of $\mathrm{Na}_{2}$ in the electronic ground state, $U_{\mathrm{X}}$ and $U_{\mathrm{A}}$ are the time evolution operators in the 
respective electronic states and $V_{\mathrm{Ax}}=f(t) \mathrm{e}^{-\mathrm{i} \omega t} \mathrm{de}-$ scribes the field/matter interaction for a pulse with shape $f(t)$ and photon energy $\omega$. We assume the projection of the transition dipole moment on the polarization vector of the laser to be equal to unity. The total ionic wavefunction for the excitation scheme indicated in fig. 1 can be written as

$|\psi(t)\rangle=\int_{0}^{\infty} \mathrm{d} E^{\prime}\left|E^{\prime}\right\rangle|+\rangle\left|\psi(t), E^{\prime}\right\rangle$.

$E^{\prime}$ corresponds to the kinetic energy of the ejected electron with wavefunction $\left|E^{\prime}\right\rangle$ and $|+\rangle$ is the electronic state of the core electrons (the ionic ground state). The vibrational wavefunction $\left|\psi(t), E^{\prime}\right\rangle$ is calculated with the second-order formula

$$
\begin{gathered}
\left|\psi(t), E^{\prime}\right\rangle=\int_{0}^{t} \mathrm{~d} t^{\prime \prime} U_{+}\left(t-t^{\prime \prime}\right) V_{+\pi}\left(t^{\prime \prime}, \omega-E^{\prime}\right) \\
\times \int_{0}^{t^{\prime \prime}} \mathrm{d} t^{\prime} U_{\pi}\left(t^{\prime \prime}-t^{\prime}\right) V_{\pi \mathrm{A}}\left(t^{\prime}, \omega\right)\left|\psi\left(t^{\prime}\right), \mathrm{A}\right\rangle .
\end{gathered}
$$

The wavefunctions in eqs. (2) and (3) depend on the delay time $\tau$ between pump and probe pulse which is not indicated explicitly. After the second laser pulse decays to zero the norm of $|\psi(t)\rangle$ remains constant and measures the total ion population.

The second pathway to ionization can be calculated in a similar way as outlined above. One first applies second-order perturbation theory to obtain the wave packet created by a two-photon transition initiated by the pump pulse and afterwards uses firstorder for the ionization step [19]. This gives another wavefunction which has to be added coherently to $|\psi(t)\rangle$ (eq. (2)) before the total population is calculated. It has been shown that the ionic population which originates from a one-photon transition from the $2^{1} \Pi_{\mathrm{g}}$ state alone does not depend on the delay time of pump and probe pulse [19]. However, the coherent sum shows strong interferences between the two ionization pathways if both pathways are of equal strength. Experimentally these strong interference patterns are not seen indicating minor contributions from the second pathway. Instead a slow modulation resulting from an incoherent superposition of the $A$ and $\Pi$ state vibrational
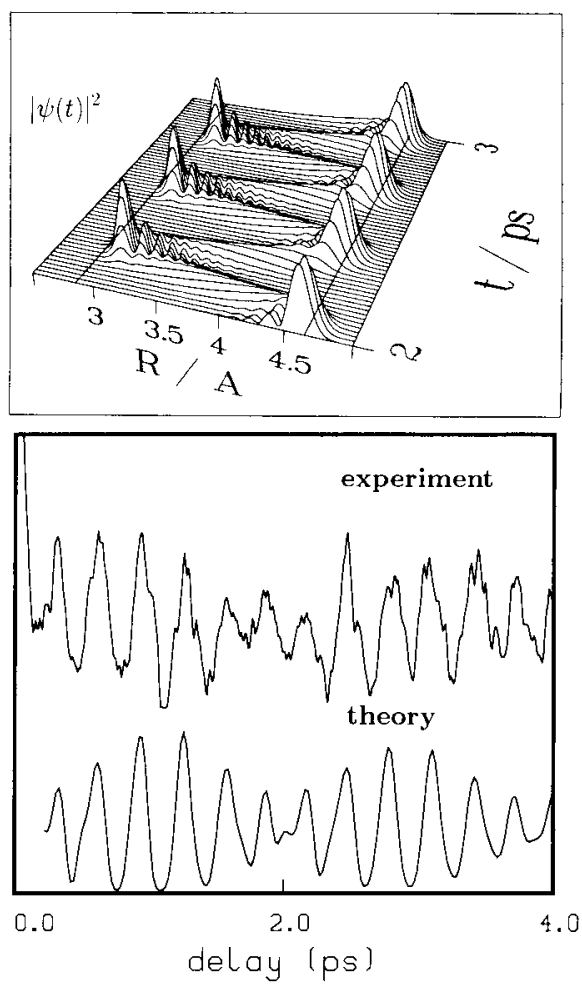

Fig. 2. Upper part: Dynamics of the vibrational wave packet in the electronic $\mathrm{A}$ state of $\mathrm{Na}_{2}$ for delay times between 2 and 3 ps. The straight lines parallel to the time axis indicate the classical turning points corresponding to the average energy of the packet. Lower part: Measured pump/probe ionization signal as a function of delay time between the laser pulses. The lower curve is the calculated total ionic population.

motion is measured, as can be taken from fig. 2 . Measurement of the $\mathrm{Na}^{+}$photofragmentation signal as a function of pump and probe pulse delay shows that there is another ionization process which reduces the probability for one-photon ionization out of the $2{ }^{1} \Pi_{\mathrm{g}}$ state when the corresponding wave packet is close to the inner turning point. This suppresses interferences since two-photon transitions from the A state occur exclusively around $3 \AA$ as indicated in fig. 1 . If one restricts the contributions from the direct photoionization out of the $2^{1} \Pi_{\mathrm{g}}$ state to higher vibrational ionic states, a nearly perfect agreement between theory and experiment is found [16]. This restriction of ionization to only higher vibrational levels $v^{+}$, which are reached from the $2^{1} \Pi_{\mathrm{g}}$ state by 
one-photon probe transitions, is applied in the calculation of the populations presented in figs. 2-4. However, the direct ionization process displayed in fig. 1 fully enters into the results.

The agreement between the above simulation and the experiment supports the evidence for yet another electronic state (a doubly excited Rydberg state) which is reached only via the outer turning point of the $2^{1} \Pi_{\mathrm{g}}$ state and participates in the ionization through electronic autoionization [15]. This is not included in the theoretical model. The integration in eq. (2) requires the discretization of the electron continuum and thus the calculation of $\left|\psi(t), E^{\prime}\right\rangle$ has to be repeated for different values of $E^{\prime}$. Besides the perturbative approach the theoretical model employs several approximations: only four electronic states are incorporated, all transition dipole moments are taken as constant and the ionization cross section is assumed to be independent of $E^{\prime}$.

The wave packet propagation is performed with the technique introduced by Feit and Fleck [20,21] and the time integrals of eqs. (1) and (3) are evaluated as outlined in ref. [22]. Alternatively one could use semiclassical methods to describe the vibrational molecular motion [23]. Throughout the calculation Gaussian shape functions $f(t)$ with a width of $70 \mathrm{fs}$ (fwhm) and a central wavelength of $618 \mathrm{~nm}$ were used.

\section{Results}

Fig. 2 compares the experimental pump/probe ionization signal and the calculated ionic population for delay times up to $4 \mathrm{ps}$. The early times when pump and probe pulse overlap are not considered in the calculation. The overall agreement between theory and measurement is excellent. Both curves show strong oscillations with a period of $\approx 308 \mathrm{fs}$. This corresponds exactly to the average vibrational splitting $\Delta E_{v^{\prime}}=107.4 \mathrm{~cm}^{-1}\left(v^{\prime}=13,14\right)[24]$ of the eigenstates coherently excited by the laser pulse. The upper part of fig. 2 illustrates the wave packet dynamics in the electronic A state. One clearly sees the oscillatory motion between the two classical turning points for the average energy of the wave packet. At times when the bond length is large, i.e. the wave packet is located at larger distances, one finds a min- imum in the ionization signal. This confirms what was said in section 1, namely that ions are built preferentialy if the packet is close to the inner turning point. The transient spectrum in fig. 2 illustrates that spreading has not yet become important.

The envelope intensity modulation of the signal in the given time interval is due to the influence of the second ionization process and reflects the oscillatory motion of another vibrational wave packet in the $2{ }^{1} \Pi_{\mathrm{g}}$ state having a classical period of $\approx 375 \mathrm{fs}$ $[15,19]$.

Spreading will become important at longer times when the influence of the anharmonicity of the potential changes the observed and calculated wave packet dynamics. This can be seen in fig. 3, which shows the ion signal, the calculated ionic population and the wave packet in an interval around $30 \mathrm{ps}$ delay time. The upper part of fig. 3 shows that the wave packet fills the whole classically allowed region and
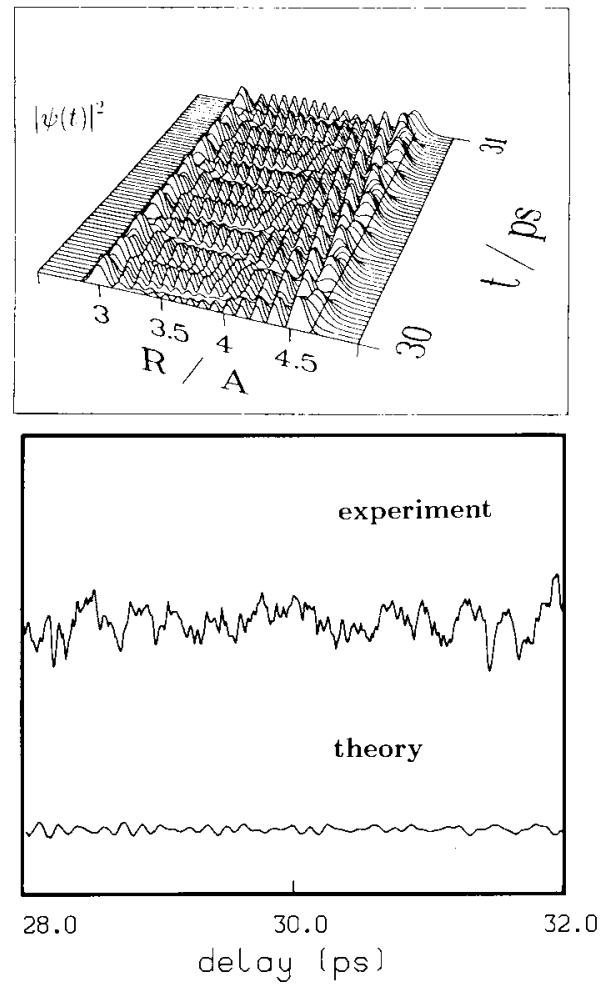

Fig. 3. The dynamics of the vibrational wave packet is shown in the upper part. The lower part of the figure contains the observed and calculated ion signal at delay times around 30 ps showing the decay of the wave packet. 
at any instant of time one finds a probability density in the inner potential region so that the transition to the ion may take place permanently. Consequently the signal has lost its periodic intensity variation and exhibits now somehow irregular fluctuations. The same is seen in the calculated ionic population.

For a non-harmonic potential the energy splitting $\Delta(v+1, v)=E_{v+1}-E_{v}$ varies as a function of $v$. Thus the classical oscillation periods $T(v+1, v)=2 \pi \hbar$ / $\Delta(v+1, v)$ will change as well with the vibrational quantum number $v$. For three levels and the assumption that $T(v+1, v)>T(v, v-1)$ the phase of the wavefunction will resemble the one for time $t=0$ if $k T(v+1, v)=(k+1) T(v, v-1)$, where $k$ counts the number of periods which have passed. This defines the recurrence time

$T_{\mathrm{rec}}=\frac{T(v+1, v) T(v, v-1)}{T(v+1, v)-T(v, v-1)}$.

For a more general discussion see ref. [5]. In the present example of the A state wave packet motion one finds a recurrence time of $T_{\mathrm{rec}} \approx 47 \mathrm{ps}$. Fig. 4 (upper part) shows that the wave packet for delay times of 47 ps is localized like it is for short times and that it again moves periodically back and forth in the bound state potential. This is beautifully seen in the observed signal (lower part of fig. 4) and in the calculated ionic population. The oscillations in the theoretical curve are shifted against the oscillations seen experimentally. The reason for this may be that the calculations are performed for zero rotational angular momentum while there is a thermal population of different rotational states which participate in the experiment. This results in a slightly different average oscillation period of the wave packet. The choice of a Gaussian envelope function for the laser pulses which has only approximately the spectral bandwidth of the real laser pulse may also contribute to that shift. If the observed and calculated periods differs by only $1 \mathrm{fs}$, a total time shift of $\approx 150$ fs has accumulated after $47 \mathrm{ps}$.

In summary, we have studied the long time behaviour of a vibrational wave packet in a bound state manifold. The spreading and recurrence of the wave packet is directly reflected in the measured pump/ probe ionization signal. Time dependent quantum calculations are performed and agree with the observed features.
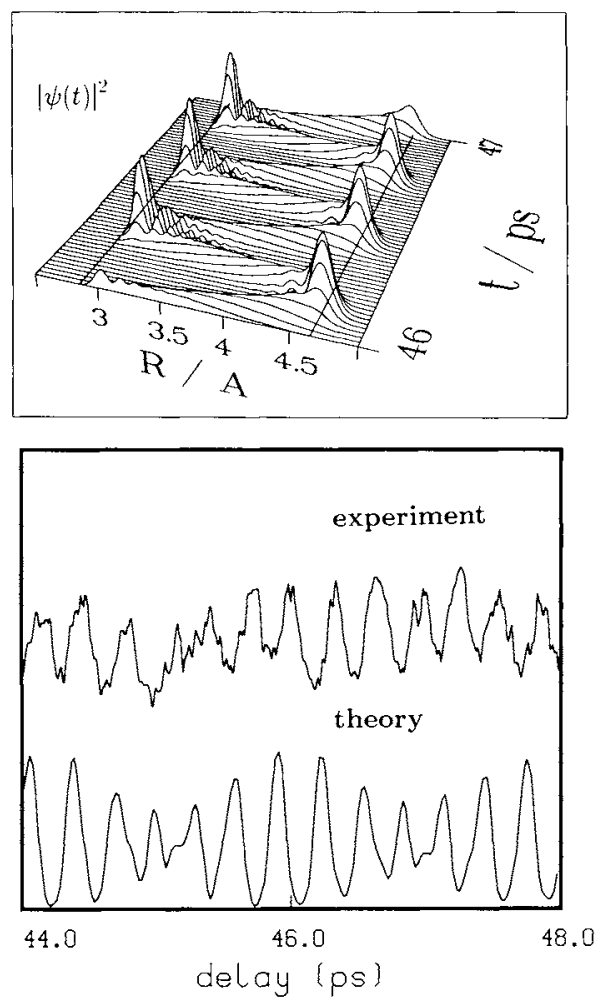

Fig. 4. The upper part shows the dynamics of the vibrational wave packet. The observed and calculated ion signal which is shown in the lower part for delay times around 47 ps clearly demonstrates the complete revival of the wave packet.

Investigations of the dynamics of Rydberg wave packets showed "fractional revivals" [6-8]. They occur at fractions of the revival time when the packet has split into two or more separate parts. More explicitly one finds for ratios $p / q$ that at times

$T_{\mathrm{rev}, p / q}=(p / q) T_{\mathrm{rev}}$

the original wave packet has split into $q$ smaller packets moving classically with the same classical period [5] but shifted against each other. This effect has not yet been seen in vibrational wave packet studies and in the present experiment. Since the calculations predict the occurrence of at least a fractional recurrence for a ratio of $p / q=1 / 2$ (around $23.5 \mathrm{ps}$ ) additional experiments with increased time resolution are currently in progress.

In the future one would like to study systems with more degrees of freedom, and the question will be if any kind of recurrence can be seen when different 
basic molecular modes are coupled to each other.

\section{Acknowledgement}

This work has been financially supported by the Deutsche Forschungsgemeinschaft within the SFB 276, TP C3 and A4.

\section{References}

[1] L.R. Khundkar and A.H. Zewail, Ann. Rev. Phys. Chem. 41 (1990) 15;

A.H. Zewail, Faraday Discussions Chem. Soc. 91 (1991) 207.

[2] T. Baumert, M. Grosser, R. Thalweiser and G. Gerber, Phys. Rev. Letters 67 (1991) 3753.

[3] M. Gruebele, G. Roberts, M. Dantus, R.M. Bowman and A.H. Zewail, Chem. Phys. Letters 166 (1990) 459.

[4] M.H.M. Janssen, R.M. Bowman and A.H. Zewail, Chem. Phys. Letters 172 (1990) 99.

[5] I.Sh. Averbukh and N.F. Perelman, Phys. Letters A 139 (1989) 449.

[6] A. ten Wolde, L.D. Noordam, A. Lagendijk and H.B. van Linden van den Heuvel, Phys. Rev. A 40 (1989) 485.

[7] J.A. Yeazell, M. Mallalieu and C.R. Stroud, Phys. Rev. Letters 64 (1990) 2007.
[8] D.R. Meacher, P.E. Meyler, I.G. Hughes and P. Ewart, J. Phys. B 24 ( 1991 ) L63.

[9] R.M. Bowman, M. Dantus and A.H. Zewail, Chem. Phys. Letters 161 (1989) 297.

[10] M.J. Rosker, T.S. Rose and A.H. Zewail, Chem. Phys. Letters 146 (1988) 175.

[11] T.S. Rose, M.J. Rosker and A.H. Zewail, J. Chem. Phys. 91 (1989) 7415 .

[12] P. Cong, A. Mokhtari and A.H. Zewail, Chem. Phys. Letters 172 (1990) 109.

[13] S. Chapman and M.S. Child, J. Phys. Chem. 95 (1991) 578.

[14] Ch. Meier, V. Engel and J.S. Briggs, J. Chem. Phys. 95 (1991) 7337.

[15] T. Baumert, B. Bühler, M. Grosser, V. Weiss and G. Gerber, J. Phys. Chem. 95 (1991) 8103.

[16] T. Baumert, G. Gerber and V. Engel, J. Chem. Phys., to be published.

[17] T. Baumert, R. Thalweiser, V. Weiss and G. Gerber, Z. Phys. $D$, to be published.

[18] J.A. Valdmanis and R.L. Fork, IEEE J. Quantum Electron. QE-22 (1986) 112.

[19] V. Engel, Chem. Phys. Letters 178 (1991) 130.

[20] J.A. Fleck, J.R. Morris and M.D. Feit, Appl. Phys. 10 (1976) 129.

[21] M.D. Feit, J.A. Fleck and A. Steiger, J. Comput. Phys. 47 (1982) 412.

[22] V. Engel, Computer Phys. Commun. 63 (1991) 228.

[23] W.T. Strunz, G. Alber and J.S. Briggs, J. Phys. B (1992), in press.

[24] G. Gerber and R. Möller, Chem. Phys. Letters 113 (1985) 546. 